\title{
Hybrid Massive MIMO Unlicensed Transmission with 1-Bit Quantization
}

\author{
Ang Li and Christos Masouros \\ Dept. of Electronic and Electrical Eng., University College London, London, U.K. \\ Email: \{ang.li.14, c.masouros\}@ucl.ac.uk
}

\begin{abstract}
We study the downlink beamforming schemes for the unlicensed wireless transmission in the cognitive radio (CR) Z-channel, where a massive multiple-input-multiple-output (MIMO) secondary base station (SBS) is considered with limited radio frequency (RF) chains and low-cost 1-bit digital-toanalog converters (DACs). In this paper, we design the analog beamformer based on the singular value decomposition (SVD) of the channel, and for the digital beamforming, instead of designing a linear transmit beamforming matrix, we propose to directly design the transmit signals by exploiting the concept of constructive interference, where the interference from the SBS to the primary users (PUs) is also taken into consideration. The optimization problem is then formulated based on the geometry of the modulation constellations and is shown to be non-convex. We then propose to solve the problem in two steps, where we firstly relax the constraint of 1-bit DACs, followed by the normalization in each entry of the baseband signals to satisfy the 1-bit DAC transmission. Numerical results show that the proposed scheme achieves an improved performance over the conventional downlink CR beamforming for the massive MIMO SBS with the hybrid structure and 1-bit DACs.
\end{abstract}

Index Terms-Massive MIMO, 1-bit quantization, downlink, constructive interference, cognitive radio.

\section{INTRODUCTION}

Cognitive radio (CR) that enables dynamic spectrum sharing has been widely acknowledged as a promising technique to improve the radio resource utilization efficiency and the spectral efficiency. The key idea of CR is to allow the utilization of the licensed spectrum by the secondary users (SUs) operating in the unlicensed spectrum [1]-[3]. In the underlay CR networks, the primary users (PUs) in the licensed band have the highest priority for the spectrum resource without the need of awareness whether there exist SUs in the CR network, while the secondary network can gain access to the licensed spectrum as long as the interference from the secondary base station (SBS) to the PUs is below a predefined threshold [2]. When multiple-input multiple-output (MIMO) systems are considered for CR networks, a fundamental problem is to design the downlink beamforming schemes for SBS to guarantee the quality of service (QoS) for the PUs, while enable the opportunistic transmission of the unlicensed SUs. Since then, transmit beamforming for CR networks has been a popular research topic, and some existing beamforming schemes targeting at signal-to-interference-plusnoise ratio (SINR) balancing, power minimization, and interference exploitation for the underlay $\mathrm{CR}$ networks have been proposed, which can be found in [4]-[8].
Towards the fifth generation (5G) and future wireless communication standards, massive MIMO techniques have become increasingly popular [9], which can greatly improve the spectral efficiency of the wireless networks with the knowledge of the channel state information (CSI). However, the consequent increasing hardware complexity and power consumption due to the increase in the number of antennas have hindered its application in practice. One promising technique is to employ the hybrid structures [10]-[13], where the power consumption is reduced dramatically by reducing the number of radio frequency (RF) chains. For the hybrid beamforming structure, the beamforming is divided into the analog domain and digital domain, where phase shifters are applied to provide highdimensional phase-only controls in the analog domain, while in the digital domain low-dimensional digital processing is applied to manage the multi-stream interference. The hybrid beamforming scheme for single-use transmission has been studied in [10], and then extended to the case of multi-user transmission in [11][12].

In addition to the hybrid structures, another potential technique that has recently received research attention tries to reduce the power consumption per RF chain, which is achieved by employing very low-resolution digital-to-analog converters (DACs) instead of high-precision DACs. It is known that the power consumption of DACs grows exponentially with the increase in the quantization precision [14], and therefore the application of 1-bit DACs can greatly reduce the power consumption for each RF chain and consequently the total power consumption of the BS. Moreover, the output signals for 1-bit DACs are equivalent to constant-envelope symbols from a QPSK constellation, which enables the use of low-cost power amplifiers (PAs) and can further reduce the hardware complexity. Due to the above benefits, downlink beamforming schemes for massive MIMO with 1-bit DACs have been proposed [15][19]. While there have been studies on the topics of both hybrid structures and 1-bit DACs, the combination of hybrid structure with 1-bit DACs for the downlink transmission and its application to CR networks has not yet been reported in the existing literature.

Therefore in this paper, we study the downlink beamforming schemes for the underlay CR Z-channel, where a massive MIMO SBS with the hybrid structure and 1-bit DACs is considered. In the proposed scheme, we employ the channeldependent analog beamforming scheme that is based on the singular value decomposition (SVD). In the digital domain, 


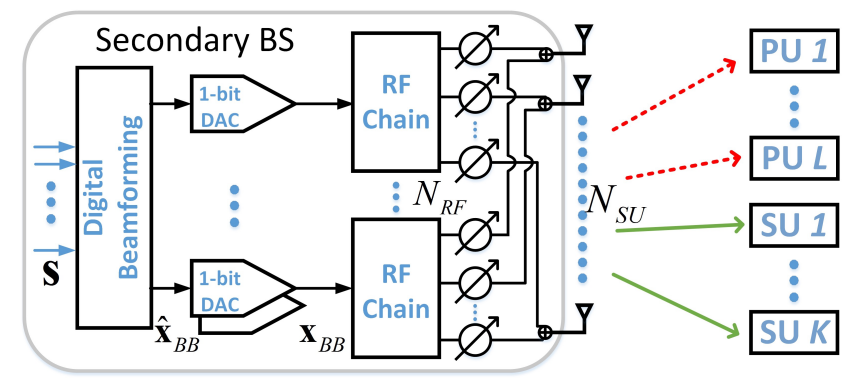

Fig. 1: Cognitive radio network topology with $K$ SUs and $L$ PUs, and hybrid 1-bit massive MIMO SBS

due to the application of 1-bit DACs, the performance of conventional digital beamforming schemes can be greatly affected due to the coarse quantization. Therefore, instead of designing the beamforming matrix, we propose to directly obtain the low-dimensional signal vector by exploiting the constructive interference (CI) [20]-[23]. The linear beamforming optimization for CI was firstly proposed in [21], and CIbased beamforming schemes have been further studied in [22], [23], where the optimization problem is formulated based on the geometry of the modulation constellations. In this paper, due to the constraint on the output signals of 1-bit DACs, the resulting optimization problem is not convex. We then propose to solve this problem in two steps, where in the first step the strict constant modulus constraint on the output signals of 1-bit DACs is relaxed, such that the optimization problem becomes convex and can be efficiently solved. Then in the second step, we apply an element-wise normalization on the signal vector obtained by the relaxed optimization problem to meet the constraint of 1-bit DACs. The numerical results show that the proposed scheme can achieve an improved performance over the conventional downlink $\mathrm{CR}$ beamforming for the considered scenario, when the massive MIMO SBS employs the hybrid structure and 1-bit DACs. Another important observation is that the hybrid structures with 1-bit DACs require a larger number of RF chains to combat the loss by quantization and achieve a satisfactory performance.

Notations: $a, \mathbf{a}$, and $\mathbf{A}$ denote scalar, vector and matrix, respectively. $(\cdot)^{T}$ and $(\cdot)^{H}$ denote transposition and conjugate transposition, respectively. $\|\cdot\|$ denotes the Frobenius norm, $|\cdot|$ denotes the modulus, and $\mathcal{C}^{n \times n}$ represents an $n \times n$ matrix in the complex set. $\Re(\cdot)$ and $\Im(\cdot)$ denote the real and imaginary part of a complex number, respectively.

\section{System Model and Conventional CR BEAMFORMING}

We consider a single-cell single-carrier downlink CR system with Z-channel, where the SBS employs massive MIMO with the hybrid structure and 1-bit DACs, as depicted in Fig. 1. We denote $N_{S U}$ and $N_{R F}$ as the number of transmit antennas and RF chains, and $L$ and $K$ as the total number of singleantenna PUs and SUs in the CR network respectively, where we have assumed $N_{t} \gg N_{R F}>K$. The considered Z-channel has been introduced in [24], where the interference from the SBS to the PUs is considered, while the interference from the primary BS to the SUs is assumed negligible. Such scenarios is most suitable when the cognitive network is far from the primary BS. Our focus in this paper is the beamforming design at the transmitter, and perfect CSI is assumed throughout the paper. We denote the transmit signal vector from SBS as $\mathbf{s} \in$ $\mathcal{C}^{K \times 1}$, which in line with the closely related literature [15][19] is assumed to be from a normalized QPSK constellation, and the proposed scheme in this paper trivially applies to other PSK and QAM modulations.

For the hybrid structure, we denote $\mathbf{F}_{R F} \in \mathcal{C}^{N_{S U} \times N_{R F}}$ as the analog beamformer implemented with phase shifters in the analog domain, and each entry of $\mathbf{F}_{R F}$ is of constant modulus. When a fully-connected RF structure is considered [10], as assumed in this paper and shown in Fig. 1, $\mathbf{F}_{R F}$ can be expressed as

$$
\mathbf{F}_{R F}=\left[\mathbf{f}_{1}, \mathbf{f}_{2}, \ldots, \mathbf{f}_{N_{R F}}\right],
$$

where each $\mathbf{f}_{k} \in \mathcal{C}^{N_{S U} \times 1}$. In this paper, we normalize each entry of $\mathbf{F}_{R F}$ to satisfy

$$
\left|\mathbf{f}_{k}(m)\right|=1 / N_{S U}, \forall m \in\left\{1,2, \ldots, N_{S U}\right\} .
$$

At the transmitter side, we denote the unquantized signal vector in the digital domain as $\hat{\mathbf{x}}_{B B} \in \mathcal{C}^{N_{R F} \times 1}$ that is formed based on $\mathbf{s}$, and therefore the signal vector $\hat{\mathbf{x}}_{B B}$ can be expressed as

$$
\hat{\mathbf{x}}_{B B}=\mathcal{B}(\mathbf{s}),
$$

where $\mathcal{B}$ denotes a general linear/non-linear mapping scheme dependent on $\mathrm{s}$. When a conventional downlink CR beamforming with infinite-precision DACs is considered for the hybrid structure, $\mathcal{B}$ is a linear mapping matrix and is commonly denoted as a beamforming matrix $\mathbf{W}=\left[\mathbf{w}_{1}, \mathbf{w}_{2}, \ldots, \mathbf{w}_{K}\right]$. In this case, $\hat{\mathbf{x}}_{B B}=\mathbf{W}$ s and the received signal for the $k$-th SU is obtained as

$$
y_{k}=\mathbf{h}_{k} \mathbf{F}_{R F} \mathbf{W} \mathbf{s}+n_{k}=\mathbf{h}_{k} \mathbf{F}_{R F} \mathbf{w}_{k} s_{k}+\mathbf{h}_{k} \mathbf{F}_{R F} \sum_{j \neq k} \mathbf{w}_{j} s_{j}+n_{k},
$$

where $\mathbf{h}_{k} \in \mathcal{C}^{1 \times N_{S U}}$ denotes the flat-fading Rayleigh channel vector from SBS to the $k$-th SU, and $n_{k}$ is the circular symmetric Gaussian distributed additive noise with zero mean and variance $\sigma^{2}$. Then, the received SINR can be expressed as

$$
\operatorname{SINR}_{k}=\frac{\left|\mathbf{h}_{k} \mathbf{F}_{R F} \mathbf{w}_{k}\right|^{2}}{\sum_{j \neq k}\left|\mathbf{h}_{k} \mathbf{F}_{R F} \mathbf{w}_{j}\right|^{2}+\sigma^{2}} .
$$


The interference from the SBS to the $l$-th PU can be obtained as

$$
\mathrm{I}_{l}=\sum_{k=1}^{K}\left|\mathbf{g}_{l} \mathbf{F}_{R F} \mathbf{w}_{k}\right|^{2},
$$

where $\mathrm{g}_{l}$ denotes the channel vector between SBS and $l$-th PU. The conventional downlink SINR balancing CR beamforming problem for the hybrid structure can then be formulated as

$$
\begin{array}{ll}
\mathcal{P}_{0}: & \max _{\gamma, \mathbf{w}_{k}} \gamma \\
\text { s.t. } & \operatorname{SINR}_{k} \geq \gamma, \forall k \in \mathcal{K} \\
& \mathrm{I}_{l} \leq \Gamma_{l}, \forall l \in \mathcal{L} \\
& \sum_{k=1}^{K}\left\|\mathbf{w}_{i}\right\|^{2} \leq P
\end{array}
$$

where $\gamma$ is an introduced variable, $\mathcal{K}=\{1,2, \ldots, K\}, \mathcal{L}=$ $\{1,2, \ldots, L\}$, and $\Gamma_{l}$ is the maximum interference allowed by the SBS for the $l$-th PU, which is also known as the interference temperature. $P$ is the maximum transmit power at the SBS. The problem $\mathcal{P}_{0}$ is feasible if the interference and power constraint have a non-zero feasible point. When only the hybrid structure is employed at the SBS, the hybrid downlink CR beamforming $\mathcal{P}_{0}$ can be solved by considering the effective channel $\hat{\mathbf{h}}_{k}=\mathbf{h}_{k} \mathbf{F}_{R F}, \hat{\mathbf{g}}_{l}=\mathbf{g}_{l} \mathbf{F}_{R F}$ and following the bisection method or iterative algorithms for the conventional CR downlink beamforming in [3]-[5].

When the hybrid structure and 1-bit DACs are both employed at the SBS, the output signal vector $\mathbf{x}_{B B}$ of the 1-bit DACs is obtained as

$$
\mathbf{x}_{B B}=\mathcal{Q}\left(\hat{\mathbf{x}}_{B B}\right),
$$

where $\mathcal{Q}$ denotes an element-wise 1-bit quantization in the real and imaginary part of $\hat{\mathbf{x}}_{B B}$. Each element in the output signal vector $\mathbf{x}_{B B}$ of 1-bit DACs therefore belongs to the set $\left\{ \pm \frac{1}{\sqrt{2}} \pm \frac{1}{\sqrt{2}} j\right\}$. In this case, we can express the received signal at the $k$-th $\mathrm{SU}$ as

$$
y_{k}=\mathbf{h}_{k} \mathbf{x}_{T}+n_{k}=\mathbf{h}_{k} \mathbf{F}_{R F} \mathbf{x}_{B B}+n_{k} .
$$

When 1-bit DACs are employed and the quantization is considered, the formulation of hybrid downlink CR beamforming $\mathcal{P}_{0}$ is no longer valid, because 1 ) it is difficult to calculate the exact expression of the received SINR; 2) it is difficult to optimize the beamforming vectors that satisfy the output signal constraint on the 1-bit DACs, which leads to the following approach based on the CI formulation.

\section{HyBRID TRANSMISSION SCHEME BASED ON CONSTRUCTIVE INTERFERENCE}

In this section the proposed hybrid transmission scheme is introduced, where we design the analog beamformer solely dependent on the channel variations. This design has the following advantages: 1) The low-dimensional digital beamformer can be designed based on the effective channel expression, and there is no need to iteratively update the analog beamformer and the digital beamformer; 2 ) the phase shifters can operate on a frame-by-frame basis instead of symbolby-symbol, and only need to change their phases when the channel varies, which is easier to be implemented in practice. As for the digital beamforming, a symbol-by-symbol design is still required since the output signals that need to satisfy the output constraint on 1-bit DACs are dependent on the data symbols, which is similar to existing schemes for 1-bit DACs [18][19]. It is this aspect of the transmission that allows us to observe interference from an instantaneous point of view, and exploit it constructively.

\section{A. Analog Beamforming Design based on SVD}

For simplicity the analog beamformer is designed based on SVD, which is only dependent on the channel. We firstly denote the channel matrix from SBS to SUs in a compact form as $\mathbf{H}=\left[\mathbf{h}_{1}^{T}, \mathbf{h}_{2}^{T}, \ldots, \mathbf{h}_{K}^{T}\right]^{T}$, and we then express the SVD of $\mathbf{H}$ as

$$
\mathbf{H}=\mathbf{U} \boldsymbol{\Lambda} \mathbf{V}^{H},
$$

where $\mathbf{U}$ and $\mathbf{V}=\left[\mathbf{v}_{1}, \mathbf{v}_{2}, \ldots, \mathbf{v}_{N_{S U}}\right]$ are unitary matrices that contain the left- and right-singular vectors, and $\mathbf{V} \in$ $\mathcal{C}^{N_{S U} \times N_{S U}}$. Then, we pick the first $N_{R F}$ columns of $\mathbf{V}$ and perform an element-wise normalization, and these column vectors after normalization satisfy the constant-modulus constraint of the phase shifters and are selected as the analog beamformers, expressed as

$$
\mathbf{f}_{k}(m)=\frac{1}{N_{S U}} e^{j \cdot \phi_{m}}, \forall m \in\left\{1,2, \ldots, N_{S U}\right\},
$$

where $\phi_{m}$ is the phase of the $m$-th entry in $\mathbf{v}_{k}$. The above ana$\log$ beamforming scheme is designed for the fully-connected structure that is assumed in this paper, and the extension to partially-connected structures [12], [13] is trivial. Moreover, other analog schemes such as discrete Fourier transform (DFT) codebooks or matched filtering [25] can also be efficiently applied upon the digital beamforming scheme introduced in the following.

\section{B. Constructive Interference based Digital Beamforming}

Thanks to the fact that the analog beamformer is solely dependent on the channel matrix, the low-dimensional digital beamforming scheme can be designed in an efficient way. We firstly express the equivalent user-to-RF channel as

$$
\mathbf{H}_{e}=\mathbf{H F}_{R F},
$$

where $\mathbf{H}_{e} \in \mathcal{C}^{K \times N_{R F}}$. based on which we design the digital scheme. For a massive MIMO system, it is commonly known that the channel matrix is near-orthogonal [9]. Nevertheless, with the hybrid beamforming structure, the resulting equivalent user-to-RF channel $\mathbf{H}_{e}$ is no longer near-orthogonal, which enables the low-dimensional digital beamforming design based on CI.

$\mathrm{CI}$ is defined as the interference that pushes the received symbols away from the detection thresholds of the modulation constellation so that a better detection performance can be obtained [20]-[23]. The exploitation of the CI is firstly introduced in [20] to improve the performance of ZF beamforming 


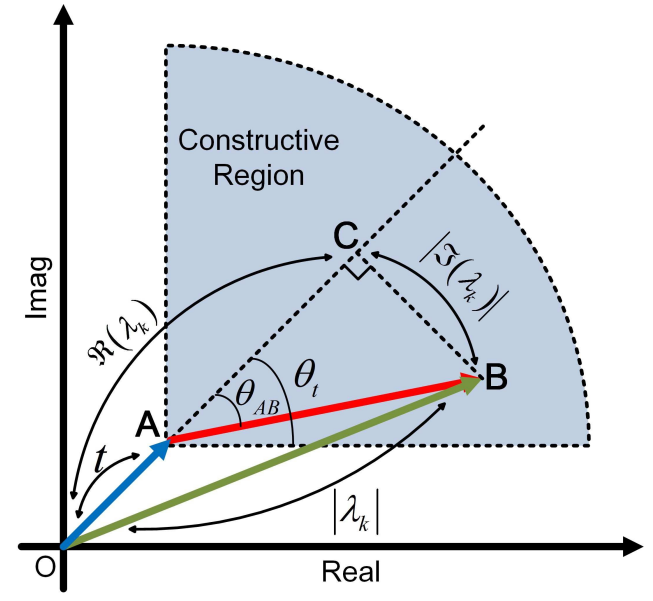

Fig. 2: Constructive region for one quarter of QPSK

scheme, where the angles of the interfering signals are controlled and strictly rotated to the phase of the desired symbols. Further studies in [23][22] have shown that the interfering signals may not necessarily be strictly aligned to the phase of the desired symbols, and additional performance gains can be obtained as long as the resulting interfered signals are located in the constructive region, within which the distance to the decision thresholds is increased. While we focus on the QPSK modulation in this paper, the extension to other PSK and QAM modulations are applicable, and we kindly refer the readers to [26][27] for a detailed description.

For the digital beamforming design, we firstly decompose the equivalent channel $\mathbf{H}_{e}$ into

$$
\mathbf{H}_{e}=\left[\hat{\mathbf{h}}_{1}^{T}, \hat{\mathbf{h}}_{2}^{T}, \ldots, \hat{\mathbf{h}}_{K}^{T}\right]^{T},
$$

where each $\hat{\mathbf{h}}_{k} \in \mathcal{C}^{1 \times N_{R F}}$ represents the equivalent $\mathrm{RF}$ channel of the $k$-th SU, and the received signal for the $k$-th $\mathrm{SU}$ is then expressed as

$$
y_{k}=\hat{\mathbf{h}}_{k} \mathbf{x}_{B B}+n_{k} .
$$

Then, we propose to exploit the CI to maximize the distance between the constructive region and the detection threshold (to improve the performance of SUs as much as possible) by directly optimizing the output signal vector $\mathbf{x}_{B B}$, while constraining the interference from the SBS to the PUs, and the optimization problem can be formulated as

$$
\begin{array}{ll}
\mathcal{P}_{1}: & \max _{\mathbf{x}_{B B}, t} t \\
\text { s.t. } & \hat{\mathbf{h}}_{k} \mathbf{x}_{B B}=\lambda_{k} s_{k}, \forall k \in \mathcal{K} \\
& \Re\left(\lambda_{k}\right)-t \geq\left|\Im\left(\lambda_{k}\right)\right|, \forall k \in \mathcal{K} \\
& x_{n} \in\left\{ \pm \frac{1}{\sqrt{2}} \pm \frac{1}{\sqrt{2}} j\right\}, \forall n \in \mathcal{N} \\
& \sum_{k=1}^{K}\left|\hat{\mathbf{g}}_{l} \mathbf{x}_{B B}\right|^{2} \leq \Gamma_{l}, \forall l \in \mathcal{L} \\
& t \geq 0
\end{array}
$$

where $\lambda_{k}$ is an introduced complex variable, $\hat{\mathrm{g}}_{l}=\mathrm{g}_{l} \mathbf{F}_{R F}$ is the effective channel between SBS and $l$-th PU, $x_{n}$ is the $n$-th entry in $\mathbf{x}_{B B}$ and $\mathcal{N}=\left\{1,2, \ldots, N_{R F}\right\}$. In $\mathcal{P}_{1}$, each $x_{n}$ needs to satisfy the output constraint for 1-bit DACs. The constructive phase constraint on each $\lambda_{k}$ in the optimization can be explained geometrically in Fig. 2 as follows, where for simplicity we focus on one quarter of the normalized QPSK constellation that corresponds to the constellation point $\left(\frac{1}{\sqrt{2}}+\frac{1}{\sqrt{2}} j\right)$. In Fig. 2, we assume $\overrightarrow{O A}=t \cdot s_{k}$ and $|\overrightarrow{O A}|=t$ is the objective function to be maximized. We assume the point ' $\mathrm{B}$ ' in Fig. 2 represents the noiseless received signal $\hat{\mathbf{h}}_{k} \mathbf{x}_{B B}$ that is located in the constructive region, and we denote $\overrightarrow{O B}=\lambda_{k} s_{k}$ with the introduced complex variable $\lambda_{k}$. Based on the geometry we can further obtain that

$$
\overrightarrow{A C}=\left[\Re\left(\lambda_{k}\right)-t\right] s_{k}, \overrightarrow{B C}=j \cdot \Im\left(\lambda_{k}\right) s_{k},
$$

where geometrically the imaginary unit ' $j$ ' denotes the angle rotation of $90^{\circ}$. Then, $\tan \theta_{A B}$ can be obtained as

$$
\tan \theta_{A B}=\frac{|C B|}{|A C|}=\frac{\left|\Im\left(\lambda_{k}\right) s_{k}\right|}{\left|\left[\Re\left(\lambda_{k}\right)-t\right] s_{k}\right|}=\frac{\left|\Im\left(\lambda_{k}\right)\right|}{\Re\left(\lambda_{k}\right)-t} .
$$

Then, to have $\overrightarrow{O B}$ located in the constructive region is equivalent to the following condition

$$
\tan \theta_{A B} \leq \tan \theta_{t} \Rightarrow\left[\Re\left(\lambda_{k}\right)-t\right] \tan \theta_{t} \geq\left|\Im\left(\lambda_{k}\right)\right|,
$$

where for $\mathcal{M}$-PSK, the angle threshold $\theta_{t}$ is given by

$$
\theta_{t}=\frac{\pi}{\mathcal{M}} \text {. }
$$

For QPSK modulation, as considered in this paper, (18) is further simplified into

$$
\Re\left(\lambda_{k}\right)-t \geq\left|\Im\left(\lambda_{k}\right)\right|,
$$

which then leads to the constructive phase constraint in $\mathcal{P}_{1}$.

Due to output constraint for the 1-bit DACs, we can observe that the optimization problem $\mathcal{P}_{1}$ is non-convex. To solve the above optimization problem in an efficient manner, in this paper we relax the strict modulus constraint for each $x_{n}$ on its real and imaginary part respectively, and the relaxed conditions for each $x_{n}$ is expressed as

$$
\left|\Re\left(x_{n}\right)\right| \leq \frac{1}{\sqrt{2}},\left|\Im\left(x_{n}\right)\right| \leq \frac{1}{\sqrt{2}}, \forall n \in \mathcal{N} .
$$

Then, the optimization problem $\mathcal{P}_{1}$ can be reformulated into its relaxation $\mathcal{P}_{2}$ as

$$
\begin{array}{ll}
\mathcal{P}_{2}: & \max _{\hat{\mathbf{x}}_{B B}, t} t \\
\text { s.t. } & \hat{\mathbf{h}}_{k} \hat{\mathbf{x}}_{B B}=\lambda_{k} s_{k}, \forall k \in \mathcal{K} \\
& \Re\left(\lambda_{k}\right)-t \geq\left|\Im\left(\lambda_{k}\right)\right|, \forall k \in \mathcal{K} \\
& \left|\Re\left(\hat{x}_{n}\right)\right| \leq \frac{1}{\sqrt{2}}, \quad\left|\Im\left(\hat{x}_{n}\right)\right| \leq \frac{1}{\sqrt{2}}, \forall n \in \mathcal{N} \\
& \sum_{k=1}^{K}\left|\hat{\mathbf{g}}_{l} \hat{\mathbf{x}}_{B B}\right|^{2} \leq \Gamma_{l}, \forall l \in \mathcal{L} \\
& t \geq 0
\end{array}
$$


where we denote the relaxed signal vector and its entry obtained by $\mathcal{P}_{2}$ as $\hat{\mathbf{x}}_{B B}$ and $\hat{x}_{n}$ respectively. It is then observed that the optimization problem $\mathcal{P}_{2}$ is a second-order cone programming (SOCP) problem and can be efficiently solved by standard convex optimization techniques. Since the relaxed constraint cannot always guarantee a strict equality on both the real and imaginary part of each $\hat{x}_{n}$, we need to force each entry of $\hat{\mathbf{x}}_{B B}$ to satisfy the constraint of the output signals with 1-bit DACs. To be specific, after we obtain the solution of $\mathcal{P}_{2}$, we proceed to impose an element-wise normalization on $\hat{\mathbf{x}}_{B B}$ for the real and imaginary part, and the resulting final solution $x_{n}$ can be obtained as

$$
x_{n}=\frac{\Re\left(\hat{x}_{n}\right)}{\sqrt{2} \cdot\left|\Re\left(\hat{x}_{n}\right)\right|}+\frac{\Im\left(\hat{x}_{n}\right)}{\sqrt{2} \cdot\left|\Im\left(\hat{x}_{n}\right)\right|} j, n \in \mathcal{N} .
$$

We note that, while we relax the modulus constraint for the output signals in the optimization problem $\mathcal{P}_{2}$, our simulation results show that most of the real part and imaginary part of the obtained $\hat{\mathbf{x}}_{B B}$ by $\mathcal{P}_{2}$ actually satisfy the modulus constraint, and only a small portion of $\hat{x}_{n}$ do not meet the constraint of 1-bit DACs, and we only need to normalize these $\hat{x}_{n}$ to satisfy the constraint. Finally, a normalization is applied so that the power constraint of the original problem is met. For completeness, the proposed constructive hybrid beamforming scheme is then summarized in Algorithm 1.

\section{Numerical Results}

In this section we present the numerical results of the proposed schemes based on Monte Carlo simulations. We assume each entry in the channel vectors is i.i.d. and follows the standard Gaussian distribution. We compare our proposed scheme ('Proposed CI') with both the linear ZF scheme ('ZF Hybrid 1-Bit' [17]) and the optimization-based hybrid CR beamforming with 1-bit DACs ('SB Hybrid 1-Bit'). 'SB Hybrid 1-Bit' is a direct combination of $\mathcal{P}_{0}$ with 1-bit DACs, whose output signal is obtained as $\mathbf{x}_{B B}=\mathcal{Q}(\mathbf{W} \mathbf{s})$, where $\mathbf{W}$

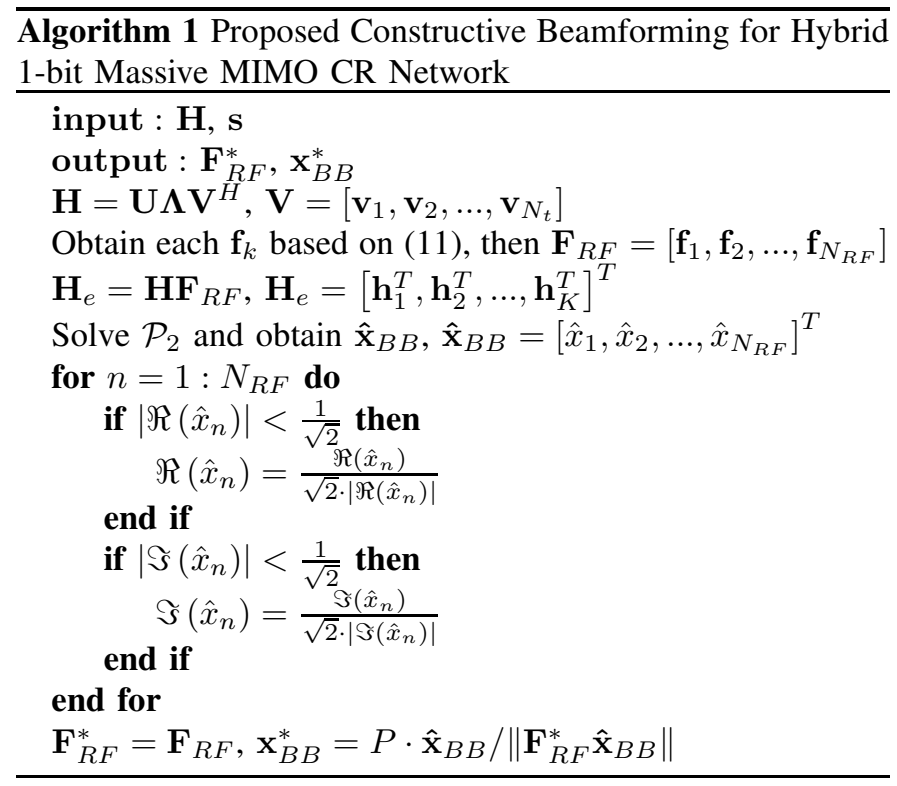

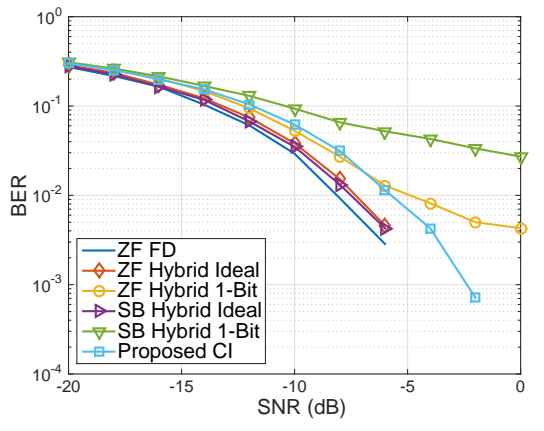

Fig. 3: BER v.s. transmit SNR, $N_{S U}=128, N_{R F}=32, K=4$, $L=2, \Gamma_{l}=0 \mathrm{dBm}, \forall l \in \mathcal{L}$

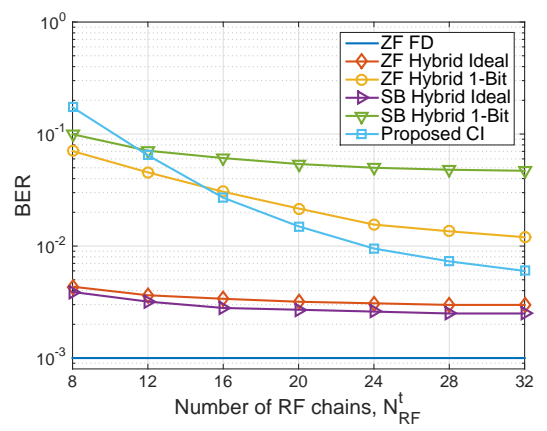

Fig. 4: BER v.s. number of RF chains $N_{R F}, N_{S U}=128, K=4$, $L=2, \mathrm{SNR}=-5 \mathrm{~dB}, \Gamma_{l}=0 \mathrm{dBm}, \forall l \in \mathcal{L}$

is obtained by solving $\mathcal{P}_{0}$. We further include the fully-digital ZF scheme ('ZF FD'), the hybrid ZF scheme with ideal DACs ('ZF Hybrid Ideal'), and solution of $\mathcal{P}_{0}$ ('SB Hybrid Ideal') as performance benchmarks.

Fig. 3 compares the bit error rate (BER) performance with the increasing transmit SNR. For ideal DACs, it is observed that 'SB Hybrid Ideal' can achieve a similar performance to 'ZF FD' and 'ZF Hybrid Ideal' for SUs, while can keep the interference to PUs below the threshold. For 1-bit DACs, it is observed that 'SB Hybrid 1-Bit' achieves the worst BER performance, while a significant performance gain can be observed for the proposed scheme. We further mention that the interference to PUs for the proposed scheme is also much smaller than the direct combination of $\mathrm{CR}$ beamforming with 1-bit DACs, which will be shown in Fig. 5.

In Fig. 4, we show the BER performance to the number of RF chains, where the transmit SNR is $-5 \mathrm{~dB}$. It is observed that the performance of fully digital scheme and hybrid scheme with ideal DACs is not significantly affected by the number of RF chains. While the performance of 'SB Hybrid 1-Bit' is not improved dramatically, we can observe a significant performance improvement for the proposed scheme with the increase in the number of RF chains.

In Fig. 5, we compare the interference from SBS to PUs with the increase in the number of RF chains. Compared to 'SB Hybrid 1-Bit' which cannot guarantee that the interference threshold is met due to the 1-bit quantization, we can observe 


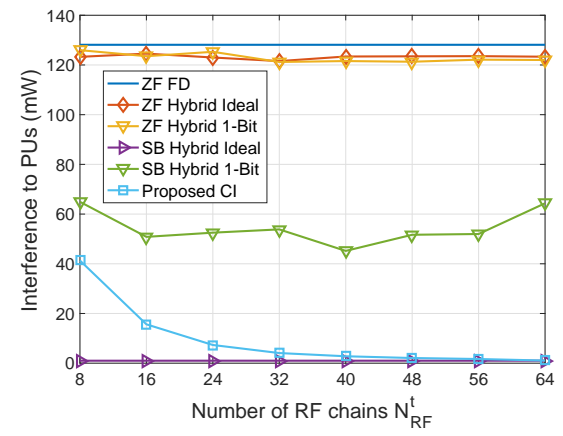

Fig. 5: Interference to PUs v.s. number of RF chains $N_{R F}$, $N_{S U}=128, K=4, L=2, \mathrm{SNR}=-5 \mathrm{~dB}, \Gamma_{l}=0 \mathrm{dBm}, \forall l \in \mathcal{L}$

a significant performance gain for the proposed scheme based on CI. We further observe that the hybrid structure with 1bit DACs requires a larger number of RF chains to combat the quantization loss by 1-bit DACs and achieve a satisfactory performance.

\section{CONCLUSION}

In this paper, we propose a CI-based transmission scheme to enable the utilization of licensed band to users operating in the unlicensed band. We employ the singular value decomposition of the channel to obtain the analog beamformer, while we directly optimize the output signals of the DACs based on the $\mathrm{CI}$ in the digital domain. A two-step approach is then proposed to solve the non-convex optimization problem. It is shown in the simulation results that the proposed scheme outperforms the case where the conventional SINR balancing CR beamforming scheme is applied to the system with 1-bit DACs.

\section{ACKNOWLEDGMENT}

This work was supported by the Royal Academy of Engineering, U.K., the Engineering and Physical Sciences Research Council (EPSRC) project EP/M014150/1, and the China Scholarship Council (CSC).

\section{REFERENCES}

[1] S. Haykin, "Cognitive Radio: Brain-Empowered Wireless Communications," IEEE J. Sel. Areas. Commun., vol. 23, no. 2, pp. 201-220, Feb. 2005.

[2] Q. Zhao and B. M. Sadler, "A Survey of Dynamic Spectrum Access," IEEE Sig. Process. Mag., vol. 24, no. 3, pp. 79-89, May 2007.

[3] T. Ratnarajah, C. Masouros, F. Khan, and M. Sellathurai, "Analytical Derivation of Multiuser Diversity Gains with Opportunistic Spectrum Sharing in CR Systems," IEEE Trans. Commun., vol. 61, no. 7, pp. 2664-2677, July 2013.

[4] I. Wajid, M. Pesavento, Y. Eldar, and D. Ciochina, "Robust Downlink Beamforming with Partial Channel State Information for Conventional and Cognitive Radio Networks," IEEE Trans. Sig. Process., vol. 61, no. 14, pp. 3656-3670, July 2013.

[5] K. L. Law, C. Masouros, and M. Pesavento, "Transmit Precoding for Interference Exploitation in the Underlay Cognitive Radio Z-Channel," IEEE Trans. Sig. Process., vol. 65, no. 14, pp. 3617-3631, July 2017.

[6] M. Alodeh, D. Spano, S. Chatzinotas, and B. Ottersten, "Peak Power Minimization in Symbol-Level Precoding for Cognitive MISO Downlink Channels," in 2016 IEEE International Conference on Digital Signal Processing (DSP), Beijing, 2016, pp. 240-244.
[7] F. Khan, C. Masouros, and T. Ratnarajah, "Interference-Driven Linear Precoding in Multiuser MISO Downlink Cognitive Radio Network," IEEE Trans. Veh. Tech., vol. 61, no. 6, pp. 2531-2543, July 2012.

[8] C. Masouros and T. Ratnarajah, "Interference as a Source of Green Signal Power in Cognitive Relay Assisted Co-Existing MIMO Wireless Transmissions," IEEE Trans. Commun., vol. 60, no. 2, pp. 525-536, Feb. 2012.

[9] F. Rusek, D. Persson, B. K. Lau, E. G. Larsson, T. L. Marzetta, O. Edfors, and F. Tufvesson, "Scaling Up MIMO: Opportunities and Challenges with Very Large Arrays," IEEE Sig. Process. Mag., vol. 30, no. 1, pp. 40-60, Jan. 2013.

[10] O. E. Ayach, S. Rajagopal, S. Abu-Surra, Z. Pi, and R. W. Heath, "Spatially Sparse Precoding in Millimeter Wave MIMO Systems," IEEE Trans. Wireless Commun., vol. 13, no. 3, pp. 1499-1513, March 2014.

[11] A. Alkhateeb, G. Leus, and R. W. Heath, "Limited Feedback Hybrid Precoding for Multi-User Millimeter Wave Systems," IEEE Trans. Wireless Commun., vol. 14, no. 11, pp. 6481-6494, Nov. 2015.

[12] A. Li and C. Masouros, "Hybrid Analog-Digital Millimeter-Wave MUMIMO Transmission with Virtual Path Selection," IEEE Commun. Lett., vol. 21, no. 2, pp. 438-441, Feb. 2017.

[13] A. Garcia, V. Venkateswaran, P. Rulikowski, and C. Masouros, "Hybrid Analog-Digital Precoding Revisited under Realistic RF Modeling," IEEE Wireless Commun. Lett., vol. 5, no. 5, pp. 528-531, Oct. 2016.

[14] R. H. Walden, "Analog-to-Digital Converter Survey and Analysis," IEEE J. Sel. Areas. Commun., vol. 17, no. 4, pp. 539-550, April 1999.

[15] A. Mezghani, R. Ghiat, and J. A. Nossek, "Transmit Processing with Low Resolution D/A-Converters," in 2009 16th IEEE International Conference on Electronics, Circuits and Systems (ICECS 2009), Yasmine Hammamet, 2009, pp. 683-686.

[16] O. B. Usman, H. Jedda, A. Mezghani, and J. A. Nossek, "MMSE Precoder for Massive MIMO Using 1-Bit Quantization," in 2016 IEEE International Conference on Acoustics, Speech and Signal Processing (ICASSP), Shanghai, 2016, pp. 3381-3385.

[17] A. K. Saxena, I. Fijalkow, and A. L. Swindlehurst, "on One-Bit Quantized ZF Precoding for the Multiuser Massive MIMO Downlink," in 2016 IEEE Sensor Array and Multichannel Signal Processing Workshop (SAM), Rio de Janerio, 2016, pp. 1-5.

[18] O. Castaneda, T. Goldstein, and C. Studer, "Pokemon: A Non-Linear Beamforming Algorithm for 1-Bit Massive MIMO," in 2017 IEEE International Conference on Acoustics, Speech and Signal Processing (ICASSP), New Orleans, 2017, pp. 1-5.

[19] A. L. Swindlehurst, A. K. Saxena, A. Mezghani, and I. Fijalkow, "Minimum Probability-of-Error Perturbation Precoding for the One-Bit Massive MIMO Downlink," in 2017 IEEE International Conference on Acoustics, Speech and Signal Processing (ICASSP), New Orleans, 2017, pp. 1-5.

[20] C. Masouros, "Correlation Rotation Linear Precoding for MIMO Broadcast Communications," IEEE Trans. Sig. Process., vol. 59, no. 1, pp. 252-262, Jan. 2011.

[21] C. Masouros, M. Sellathurai, and T. Ratnarajah, "Vector Perturbation based on Symbol Scaling for Limited Feedback MISO Downlinks," IEEE Trans. Sig. Process., vol. 62, no. 3, pp. 562-271, Feb. 2014.

[22] C. Masouros and G. Zheng, "Exploiting Known Interference as Green Signal Power for Downlink Beamforming Optimization," IEEE Trans. Sig. Process., vol. 63, no. 14, pp. 3628-3640, July 2015.

[23] M. Alodeh, S. Chatzinotas, and B. Ottersten, "Constructive Multiuser Interference in Symbol Level Precoding for the MISO Downlink Channel," IEEE Trans. Sig. Process., vol. 63, no. 9, pp. 2239-2252, May 2015.

[24] S. Vishwanath, N. Jindal, and A. Goldsmith, "The 'Z' Channel," in Global Telecommunications Conference, 2003. GLOBECOM 03, vol. 3, Dec. 2003, pp. 1726-1730.

[25] L. Liang, W. Xu, and X. Dong, "Low-Complexity Hybrid Precoding in Massive Multiuser MIMO Systems," IEEE Wireless Commun. Lett., vol. 3, no. 6, pp. 563-656, Dec. 2014.

[26] C. Masouros, T. Ratnarajah, M. Sellathurai, C. B. Papadias, and A. K. Shukla, "Known Interference in the Cellular Downlink: A Performance Limiting Factor or a Source of Green Signal Power?" IEEE Commun. Mag., vol. 51, no. 10, pp. 162-171, Oct. 2013.

[27] A. Li and C. Masouros, "Exploiting Constructive Mutual Coupling in P2P MIMO by Analog-Digital Phase Alignment," IEEE Trans. Wireless Commun., vol. 16, no. 3, pp. 1948-1962, Mar. 2017. 